\title{
Automatic Segmentation of Macular Holes in Optical Coherence Tomography Images: A review
}

\section{Odilon Linhares C. Mendes ${ }^{1}$, Abrahão R. Lucena ${ }^{2}$, Daniel R. Lucena ${ }^{2}$, Tarique S. Cavalcante ${ }^{1}$, Auzuir R. de Alexandria ${ }^{1, *}$}

\author{
${ }^{1}$ Instituto Federal do Ceará - IFCE, Campus Fortaleza, Av. Treze de Maio, 2081, Benfica, 60040-215 Fortaleza, Ceará, Brazil \\ Email: odilonlinharesmendes@gmail.com; tariquesc@gmail.com; auzuir@gmail.com \\ ${ }^{2}$ Escola Cearense de Oftalmologia, Avenida Oliveira Paiva, 1599, Cidade dos Funcionários, Fortaleza, Ceará, Brazil \\ Email: abrahaorlucena@gmail.com; danielrlucena@gmail.com \\ *Corresponding Author: Auzuir R. de Alexandria, Email: auzuir@gmail.com
}

\begin{abstract}
How to cite this paper: Mendes, O. L. C. Lucena, A. R., Lucena, D. R., Cavalcante, T. S. and Alexandria, A. R. (2019) Automatic Segmentation of Macular Holes in Optical Coherence Tomography Images: A review. Journal of Artificial Intelligence and Systems, 1, 163-185

https://doi.org/10.33969/AIS.2019.11010

Received: December 6, 2019

Accepted: December 27, 2019

Published: January 17, 2020

Copyright (c) 2019 by author(s) and Institute of Electronics and Computer. This work is licensed under the Creative Commons Attribution International License (CC BY 4.0).

http://creativecommons.org/licenses/by/4.0/

\section{(c) 9 Open Access}

\begin{abstract}
Macular holes are a blinding condition that occur due to overuse of the fovea, in which a hole alters the natural retinal structure. Optical Coherence Tomography (OCT) is a way of mapping and shaping retinal sections without physical contact and has become a powerful tool for diagnosing pathologies. This paper deals with a review of automated segmentation of macular holes in OCT images, detailing its varied possibilities. It may be considered something new, no reviews were made about the topic. The purpose of this review is to show the latest trends, through the approaches in preprocessing and segmentation. Recent studies were used to validate the research, 2011 onwards, from the Science Direct, IEEE, PubMed and Google scholar bases. The objectives, methodology, tools, database, advantages, disadvantages, validation metrics and results of the selected material are analyzed and mentioned. Based on this, techniques and their results are compared. From this, future outlook scenarios of automated segmentation of macular holes in OCT images are mentioned.
\end{abstract}

\section{Keywords}

Macular holes, Optical Coherence Tomography (OCT), Segmentation, Preprocessing, Pathology

\section{Introduction}

In the central region of the retina is the macula, and the fovea is near its center. This system allows detailed view [1]. Some macular pathologies are central serous retinopathy (CSR), age-related macular degeneration (AMD) and macular edema (ME) [2-4]. A distinct macular disease less commonly observed is called macular hole (MH) [5]. Macular holes are blinding conditions, that occur due to overuse of the fovea, in which a hole alters the natural retinal structure [6]. The $\mathrm{MH}$ pathology affects about 1 per 500 patients over 40 years [7], and for those over 55 years the number rises to 1.65 per 500 patients [8]. Medical advances make people live longer and this caused an increase number in retinal deseases $[9,10]$. 
The main effects due this pathology is the vision reduction or even total blindness [11]. It can cause negative impacts on the quality of life because the limitations of vision. The treatment or a possible surgery depends of the size and shape of the MH. Other important variables are volume, areas, diameters and height. These measures are important to assess if it is possible the hole closure [12-14]. The identification of MH in OCT images can be made through the top boundaries of the internal limiting membrane (ILM) and the retinal pigment epithelium (RPE) [15-17]. In Figure 1 is shown some important measures to analysis this pathology. MH can be full-thickness holes (FH) and pseudoholes (PH) [18]. For each situation, there is a different way of treating it [19]. The MH size is the major risk factor in case of complications in surgery [20], and it determine the approach, if just a treatment with enzymatic vitreolysis or surgery [21,22]. Surgeries has achieved good results in visual restoration of patients [23-25].
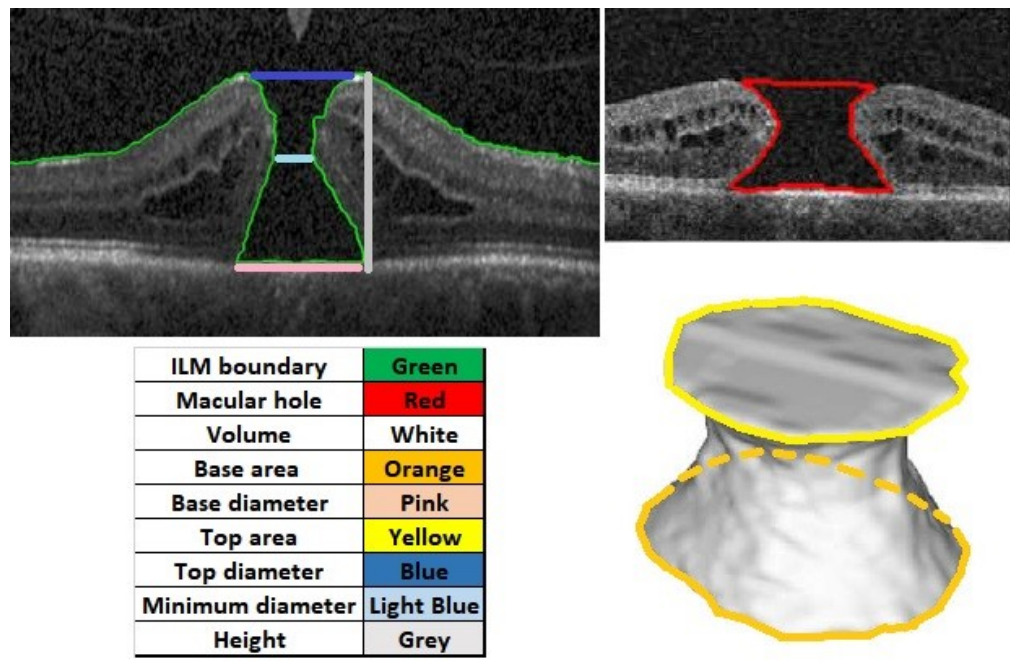

Figure 1. Some measures of MH pathology. Adapted from [26] and [27].

The OCT is a way of mapping and shaping retinal sections without physical contact and has become a powerful tool for diagnosing pathologies [28-30]. OCT provides high quality retinal images. It was first introduced in 1991 [31-33], but it appeared commercially only in 1996 [34]. Now it is standard for medical retinal analysis [35]. Various A scans (1D) build the B scan (2D) image [36], and an aggregation of B-scans constructs a 3D structure [37-39]. These images can have speckle noise, that is a leading problem [40-42], becoming hard to find out how to segment and trace boundaries without this issue compromise the results [43-45]. Shadows caused by retinal blood vessels and some pathologies on retinal structures can challenge even more the segmentation, therefore problems related to a wrong contour are more likely to occur $[46,47]$.

There are two ways to remove stains on OCT: parallel method, it preserves the resolution, improves contrast, but changes physical structure [48-50]; and serial method, it preserves the physical structure, but is more time cost [51, 52]. Cirrus HD-OCT, 3D-OCT 100 and 2000, RS-3000, Stratus OCT, DRI OCT-1 and Spectralis OCT are some examples of SDOCT devices [53, 54]. The images of OCT devices are better than those from ultrasound or magnetic-resonance imaging [55]. These images are needful before any conclusion for surgery [56-58], because they provide base to study the development of retinal diseases $[59,60]$. There are advantages using OCT: image acquisition quickly [61], great sensitivity to very low light [62] and high resolution for millimeter structures [63].

The OCT devices have their own manual segmentation application [64]. Manual segmentation of boundary layers in OCT images is essentially made by experts. This method takes a considerable time and becomes disadvantageous in studies of segmentation 
and classification [65-69]. Manual segmentation is yet the major technique of segmentation due the lack of trustworthy automated methods [70]. Thus, a fast and low cost segmentation technique is interesting to help these experts with the retina layers analysis. Automatic segmentation and diagnosis of pathologies using OCT is not an easy task, more than one pathology may affects negatively the results. For the same pathology may exist different characteristics. The MH is one example. The guaranty of an identical comparison between regions is not possible in some studies that use more than one device [71, 72].

Despite the challenges encountered in segmentation techniques of MH pathology, the motivation to produce this review is the importance of automated methods to segment OCT images and help the specialists. Besides that, the advances in research involving this theme can contribute to the total acceptance to use this technology in medical practices. This work may be a source of study for researchers, clinicians and engineers wishing to delve into MH segmentation techniques. They are together seeking improvements in retinal image analysis [73, 74]. Different parameters acquired by OCT images contribute with patient diagnosis and with academic researches [75, 76]. Computer-aided diagnosis systems (CAD) may help health professionals making conclusions because they provide information beyond the images [77-79].

A limited amount of researches has been made in this field of study. Therefore, this review can contribute to spread more researches and approaches on segmentation of $\mathrm{MH}$. The main objective of this article is to analyze the latest technologies used for segment $\mathrm{MH}$ in OCT images, through the approaches in preprocessing, segmentation and extraction of attributes. Some approaches were performed in order to automatic segment macular holes from OCT scans. As the MH varies according to its size and shape, automatic segmentation becomes more difficult.

A review of these techniques may be considered something new, no reviews were made about the topic. The survey is timely mainly due the recent increase of publications in the field. It is possible to find more works being produced from 2018 to nowadays than in other years. New technology trends, such as machine learning and deep learning have been sprayed in the last three years, as examples: [80], [81] and [82]. This work details the tasks to segment $\mathrm{MH}$ and argues the techniques step by step. For each identified approach, the database, the method and the results are discussed.

Automatic segmentation, specifically of $\mathrm{MH}$, are limited in a small quantity of 6 works and there is no review yet in this area. In order to go deeper, this review proposes to examine the main techniques of other works that uses similar techniques to segment other macular pathologies. The principles are the same and they can be applied in $\mathrm{MH}$, segmenting boundary layers from OCT images in order to obtain specific characteristics for certain macular diseases. The review of pulmonary nodule detection made by Valente [83] served as a source of inspiration and learning for this review. As the work of [83], this review seeks to find the best techniques to help with medical imaging diagnostics.

The review is divided into: Section 2 describes the datasets and research methods used in this work. Section 3 details the assessment of CV systems. Section 4 discusses the results, the key words more commonly used and analysis of the works that best match the research. Section 5 is the Discussion section, where an analysis about the advantages and disadvantages of the relevant works is made. Lastly, Section 6, the Conclusion section, summaries the results of this review.

\section{Work selection criteria}

To develop this review, some steps were necessary: (1) to carry out research of the subject from the Science Direct, IEEE, PubMed and Google scholar bases; (2) choose the works based on the adopted method; (3) synthesize the keywords, in order to ensure relevant research; and (4) evaluate each selected work.

The following logical expression used in the bases was: (segmentation OR classification 
OR detection OR "macular holes" OR MH) AND ("optical coherence tomography" OR OCT). Each base has its own search settings that require adaptation. The search went beyond the expression used, utilizing relevant works found in the references of some studied papers. It was obtained a total of 128 works. From these papers, 32 were chosen to be studied.

Each work was checked aim at divide them with respect to their application: automated segmentation of MH from OCT images (6 articles); automated segmentation of boundary layers with other pathologies in OCT images, similar approach from MH segmentation (21 articles); and segmentation approaches in classification of macular pathologies, correlated works which describe segmentation techniques before the classification step (5 articles). Although only 6 articles are directly linked to segmentation of $\mathrm{MH}$, the other works were supportive because their techniques can be transmitted to macular holes analysis.

\section{Computer Vision systems}

Diagnosis using medical images are popular trustworthy. Millions of images are produced by hospitals each year. They come from different sources like magnetic, tomography, or ultrasound. These data have features that can allow specialists diagnose pathologies [84]. The CV obtains information through image analysis. To process it, first is required to convert it into a digital image (pixels), which may vary in grayscale or mix of primary colors [85].

Computer vision ensures visual information for a given application. It is a branch of artificial intelligence (AI). Automated systems can take information of data and perform some tasks [86]. This technology is improving several areas: medicine, industry, science, military force etc [87]. There are some levels of vision: low-level vision, gathering and processing of images; intermediate-level vision, segmentation and classification; and highlevel vision, AI that generates results [88]. The CV has four phases: acquisition of data, preprocessing, segmentation and classification. Theses phases of the selected works are shown in Table 1.

\subsection{Acquisition of data}

Data is acquired by images from the OCT technique. Images of public databases are used for researches in different applications. Through these databases, researchers can make studies and comparisons between similar works [89]. From this review, most of the works used particular database only or together with some public ones. Other works did not mention the used database.

Some public databases from OCT images are: Duke dataset [90], OCT Retinal IMage Analysis 3D (OCTRIMA-3D) database [91], Mendeley Dataset [92] and Noor Eye Hospital in Tehran database [93, 94]. These databases include the following conditions: normal macula (NM), diabetic macular edema (DME), dry age-related macular degeneration (AMD), choroidal neovascularization (CNV) and Drusen. For the specific case of $\mathrm{MH}$, all of the 6 directly related works utilize particular databases. It was found just one publicly database with MH pathology in OCT scans: Optical Coherence Tomography Image Retinal Database (OCTID) [95].

The Duke database is a publicly available database of Individual spectral domain SDOCT images with 38400 B-scans with AMD and NM. This database contains their ages, and their corresponding segmentation boundaries on a $5 \mathrm{~mm}$ diameter centered at the fovea [90]. OCTRIMA-3D dataset is a publicly database that has 10 SD-OCT volume. There are raw images, manual markings of two experts and results. Speckle noise was reduced and contrast enhancement was made in these images [91, 114].

The Mendeley Dataset has data to train and test algorithms. The images are mixed in four conditions: CNV, DME, Drusen, and NM. This dataset has 5.8 Gigabyte of OCT images [92]. The Reza Rasti database is another publicly available database. It consists of NM, AMD and DME conditions. There are around 512 to 768 A-scans that together make 
Table 1. CV phases of the selected works.

\begin{tabular}{|c|c|c|c|c|}
\hline Authors & Year & Preprocessing & Segmentation & Classification \\
\hline Nasrulloh et al. [11] & 2018 & Yes & Yes & No \\
\hline Keller et al. [26] & 2016 & Yes & Yes & No \\
\hline Miri et al. [96] & 2016 & Yes & Yes & No \\
\hline Zhang et al. [5] & 2015 & Yes & Yes & No \\
\hline Xu et al. [27] & 2013 & Yes & Yes & No \\
\hline Liu et al. [19] & 2011 & Yes & Yes & Yes \\
\hline Duan et al. [43] & 2017 & Yes & Yes & No \\
\hline Sui et al. [28] & 2017 & No & Yes & No \\
\hline Hu et al. [65] & 2019 & Yes & Yes & No \\
\hline Hussain et al. [97] & 2015 & Yes & Yes & No \\
\hline ElTanboly et al. [98] & 2016 & Yes & Yes & No \\
\hline Hussain et al. [99] & 2017 & Yes & Yes & No \\
\hline Chiu et al. [100] & 2012 & Yes & Yes & No \\
\hline Novosel et al. [101] & 2017 & Yes & Yes & No \\
\hline Naz et al. [102] & 2017 & Yes & Yes & No \\
\hline Xiang et al. [103] & 2019 & Yes & Yes & No \\
\hline Hassan et al. [104] & 2018 & Yes & Yes & No \\
\hline González-López et al. [105] & 2019 & Yes & Yes & No \\
\hline Stankiewicz et al. [106] & 2017 & Yes & Yes & No \\
\hline Athira et al. [107] & 2018 & Yes & Yes & No \\
\hline Gopinath et al. [108] & 2017 & No & Yes & No \\
\hline Dodo et al. [109] & 2019 & Yes & Yes & No \\
\hline Duan et al. [110] & 2015 & Yes & Yes & No \\
\hline Lang et al. [111] & 2017 & Yes & Yes & No \\
\hline Niu et al. [112] & 2014 & Yes & Yes & No \\
\hline Rossant et al. [113] & 2015 & Yes & Yes & No \\
\hline Tian et al. [114] & 2015 & Yes & Yes & No \\
\hline Huang et al. [80] & 2019 & No & Yes & Yes \\
\hline Nath et al. [82] & 2018 & Yes & Yes & Yes \\
\hline Hassan and Hassan [81] & 2019 & Yes & Yes & Yes \\
\hline Hassan et al. [1] & 2016 & Yes & Yes & Yes \\
\hline Fang et al. [115] & 2017 & Yes & Yes & Yes \\
\hline
\end{tabular}

the B-scans $[93,94]$. The OCTID was the only publicly available database found with only cases of macular holes pathology [95]. This dataset has $102 \mathrm{~B}$-scan OCT images. There is no ground-truth or any manual segmentation made by experts to make comparisons and validate algorithms.

\subsection{Preprocessing}

Preprocessing image techniques for this review are performed in order to improve their characteristics based on the type of application to the next step, which is the segmentation of a subject in a particular region of interest (ROI). This stage is important due OCT images eventually contain inhomogeneity, speckle noise and shadows caused by retinal blood vessels [116].

The review indicates many types of preprocessing techniques: dilation and erosion [5], median filter [27, 107, 111], gaussian filter [11, 100, 101], wiener filter [11, 81, 99], binary image [26, 100], gradient image [26, 114], anisotropic difusion filter [5, 97, 99], image aligment [19, 98, 103], attenuation coefficient [101], enhanced contrast [1, 105], image flattenig [106, 114], resize the image [1, 107], edge flow [112], sparse filter [112], 
normalization [81], green channel [81], greyscale [1], morphological operations [1] and others. In Figure 2 is shown the improvements of preprocessing applied to OCT image.

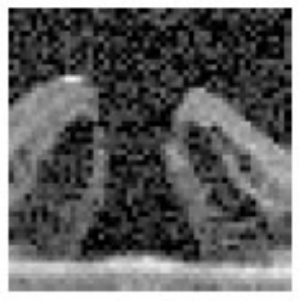

(a)

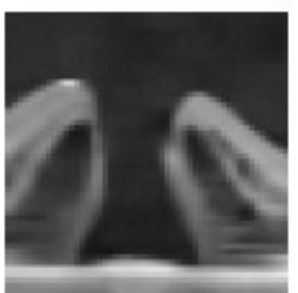

(b)

Figure 2. (a) Original OCT image and (b) denoise using the Wiener filter [11].

\subsection{Segmentation}

Segmentation is a fundamental branch of CV system that collaborates to the automated diagnosis of $\mathrm{MH}$ and other pathologies. In the case of $\mathrm{MH}$, this task is challenging because the top boundary of the ILM is irregular and nonlinear. It is not coincidence that there are few works that explore the segmentation or classification for this pathology. Some metrics can be used to validate an algorithm: accuracy, processing time and error variation.

Some segmentation techniques applied to OCT images are: snakes or active contour, it is a method used to circumvent objects in an image [105]; graph search, this technique starts from a starting point to an ending point, repeating the process, trying to find the best path [5, 26, 27]; Dijkstra shortest path search, it is an algorithm that seeks to find the shortest path between two nodes [26, 99, 107]; local gaussian distribution fitting (LGDF), local entropy defines gray level weights [11]; curvature-based surface cutting, it can be flattened into the plane with low metric distortion [11]; ReLayNet, it is an architecture used to segment retinal layers [80,117]; speed-up robust features (SURF), it is a method used in tasks like object recognition, classification etc [115]; adjusted mean arc length (AMAL), it enables to pass the load limit and turning points, and consequently to follow the post-critical equilibrium trajectories [26]; gradient vector flow (GVF), it is an algorithm that locates object edges $[96,118,119]$.

Other techniques are: multi-scale spatial pyramid (MSSP), it captures the geometry of retina at multiple scales [19]; geodesic distance method (GDM), it can locate pixels in boundaries of layers [43]; convolutional neural network $(\mathrm{CNN})$, it is pooling, which is a non-linear down-sampling $[28,108,120]$; dynamic programming (DP), it is a method that divide problems and solves each one separately $[65,100,102]$; canny edge detection, it is an algorithm that detects edges [97, 99, 104]; markov gibbs random field (MGRF), it allows to derive a global texture description by specifying local properties of textures [98]; loosely coupled level sets (LCLS), it is a technique that uses local intensity variations to segment layers [101]; structure tensor, it utilizes the gradient of a point with neighborhood to get directions of segmentation [102, 104]; Randon Forest (RF), that train the data to estimate boundary probabilities [111, 121, 122]; and OTSU algorithm, it is used to perform automatic image thresholding [102, 107, 112]. In Figure 3 is shown an example of segmentation approach applied to an OCT image, in which the top boundary of the ILM and RPE layers are highlighted. The automatic segmentation methods of $\mathrm{MH}$ or similar pathologies are divided and indicated in Table 2. 


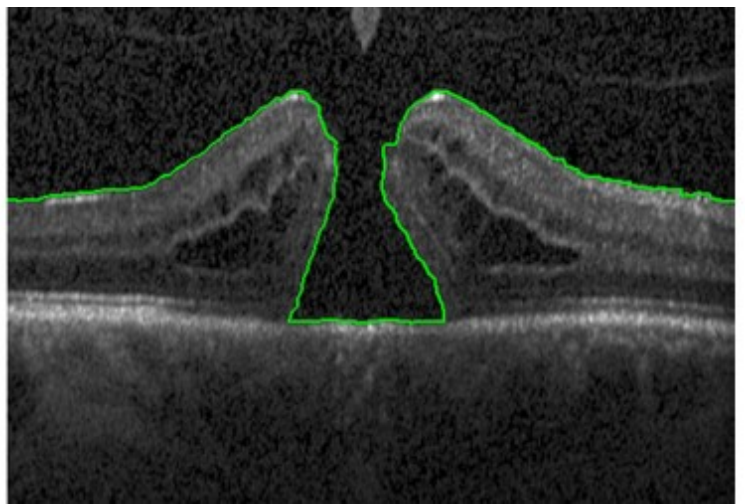

Figure 3. Segmentation of a macular hole [26].

\subsection{Classification}

Classification is based on information of the observed data. Usually are extracted features that differentiate some patterns. In the case of classification of pathologies in OCT images, the previous step, that is the segmentation, is the focus in these articles. Classification techniques can be performed in segmentation approaches too. Some works in this field combine image processing with techniques of AI [123-125]. The classification of retinal pathologies can be seen in many works [126]. Some examples in this review are: convolutional neural network (CNN) [80], k-nearest neighbour (KNN) [82, 127], suporting vector machine (SVM) [1, 81], multi-instance multilabel learning (MML-LR) [115] and adaboost classifier [5]. In Figure 4 is given an illustration of some pathologies classified based on their boundary layers.

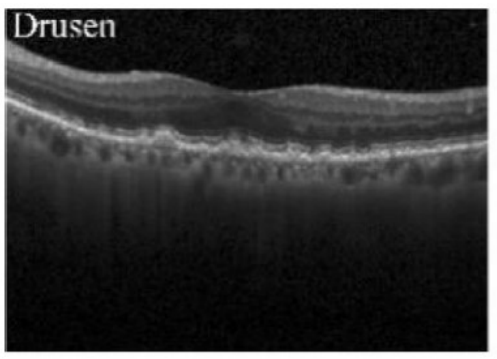

(a)

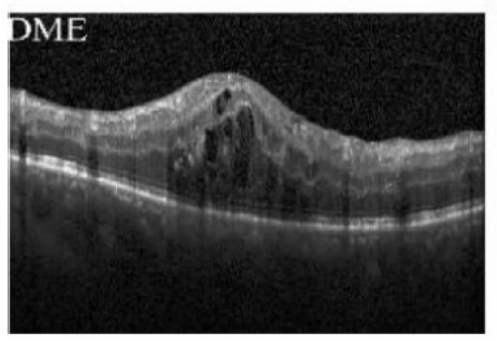

(c)

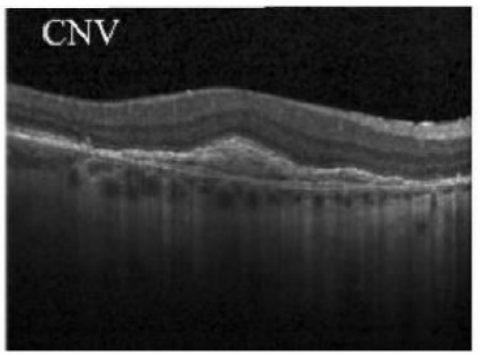

(b)

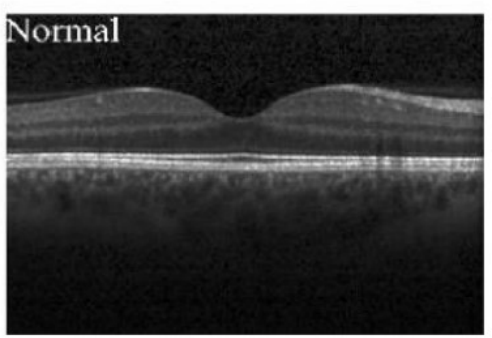

(d)

Figure 4. Some retinal pathologies [128].

\section{Selected Works}

Some metrics of validation were observed in the articles for this review and it is important to understand their meaning, they are: standard deviation (SD), it measures dispersion from the 
Table 2. Automatic segmentation of MH or similar boundaries.

\begin{tabular}{|c|c|c|c|}
\hline $\begin{array}{c}\text { Type of } \\
\text { segmentation }\end{array}$ & $\begin{array}{c}\text { Segmented } \\
\text { boundary } \\
\text { layers } \\
\end{array}$ & Authors & $\begin{array}{l}\text { Segmentation } \\
\text { technique(s) }\end{array}$ \\
\hline \multirow[t]{6}{*}{$\begin{array}{l}\text { Segmentation } \\
\text { of MH }\end{array}$} & 1 & Nasrulloh et al. [11] & $\begin{array}{l}\text { LGDF / Curvature-based } \\
\text { Surface Cutting }\end{array}$ \\
\hline & 1 & Keller et al. [26] & $\begin{array}{l}\text { AMAL / Graph Search / } \\
\text { Dijkstra }\end{array}$ \\
\hline & 1 & Miri et al. [96] & GVF / Graph Theory \\
\hline & 2 & Zhang et al. [5] & Graph Cut \\
\hline & 2 & Xu et al. [27] & MS-3D Graph Search \\
\hline & NI & Liu et al. [19] & MSSP \\
\hline \multirow{26}{*}{$\begin{array}{l}\text { Segmentation } \\
\text { of boundary } \\
\text { layers }\end{array}$} & 9 & Duan et al. [43] & GDM \\
\hline & 2 & Sui et al. [28] & CNN / Graph Search \\
\hline & 5 & Hu et al. [65] & CNN / Graph Search \\
\hline & 3 & Hussain et al. [97] & $\begin{array}{l}\text { Graph Search / Dijkstra / } \\
\text { Canny Edge }\end{array}$ \\
\hline & 12 & ElTanboly et al. [98] & MGRF \\
\hline & 4 & Hussain et al. [99] & Dijkstra / Canny Edge \\
\hline & 3 & Chiu et al. [100] & $\begin{array}{l}\text { Dijkstra / } \\
\text { Graph Theory / DP }\end{array}$ \\
\hline & 8 & Novosel et al. [101] & LCLS \\
\hline & 8 & Naz et al. [102] & $\begin{array}{l}\text { DP / Structure tensor / } \\
\text { Canny Edge / OTSU }\end{array}$ \\
\hline & 11 & Xiang et al. [103] & $\begin{array}{l}\text { RFC / Single Graph Live } \\
\text { Wire Algorithm }\end{array}$ \\
\hline & 6 & Hassan et al. [104] & $\begin{array}{l}\text { Structure tensor / } \\
\text { Canny Edge }\end{array}$ \\
\hline & 4 & González-López et al. [105] & Snakes or Active Contour \\
\hline & 2 & Stankiewicz et al. [106] & $\begin{array}{l}\text { Peak Intensity Analysis / } \\
\text { Graph Theory }\end{array}$ \\
\hline & NI & Athira et al. [107] & $\begin{array}{l}\text { Dijkstra Shortest Path / } \\
\text { OTSU }\end{array}$ \\
\hline & 8 & Gopinath et al. [108] & $\mathrm{CNN}$ \\
\hline & 9 & Dodo et al. [109] & Level Set Method \\
\hline & 12 & Duan et al. [110] & Region Growing Method \\
\hline & 8 & Lang et al. [111] & RFC / Graph Search \\
\hline & 6 & Niu et al. [112] & $\begin{array}{l}\text { OTSU / Polynomial } \\
\text { Fitting Function }\end{array}$ \\
\hline & 8 & Rossant et al. [113] & Snakes or Active contour \\
\hline & 8 & Tian et al. [114] & $\begin{array}{l}\text { Graph Search / } \\
\text { Dijkstra shortest path }\end{array}$ \\
\hline & 2 & Huang et al. [80] & ReLayNet \\
\hline & 7 & Nath et al. [82] & LCLS \\
\hline & 8 & Hassan and Hassan [81] & $\begin{array}{l}\text { Tensor Grid / OTSU / } \\
\text { Canny Edge }\end{array}$ \\
\hline & 8 & Hassan et al. [1] & OTSU / Canny Edge \\
\hline & 2 & Fang et al. [115] & SURF \\
\hline
\end{tabular}


mean of a dataset; sensitivity, or true positive rate (TPR), or recall, it indicates the amount of correctly identified actual positives; specificity, or true negative rate (TNR) indicates the amount of correctly identified actual negatives; accuracy, it indicates how close the values are measured to a target; signed error (SE), it is a sample statistic that summarizes how well a set of estimates match the quantities that they are supposed to estimate; unsigned error (UE), it is the opposite of signed error.

Other important metrics are: jaccard Index, it is used in understanding the similarities between sample sets; Dice similarity coefficient (DSC), it is a reproducibility validation metric; operator characteristic curve (AUC) or ROC curve, it evaluates the binary classifier as its discrimination threshold varies.; average deviation distance (AD) or mean absolute deviation (MAD), it is the average distance between each data point and the mean; firstorder agreement coefficient (AC1), it is the probability of agreement of evaluators; Pearson correlation, it indicates how two variables are linearly related; and signal to noise ratio (SNR), it is a comparison between desired signal and the level of background noise.

During research, many keywords were found. In Figure 5 is shown the most relevant keywords to this field of study. This section analysis the best works exploring the most recent and important approaches for automated segmentation in OCT images.

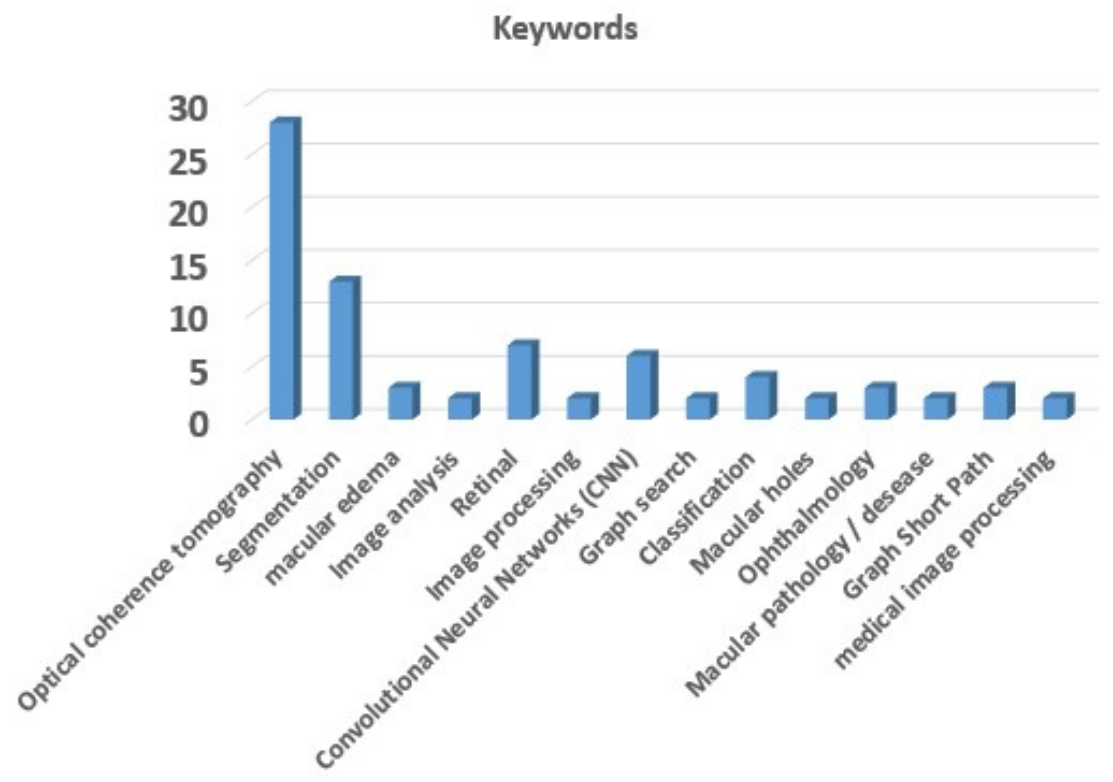

Figure 5. The best and most effective keywords.

Nasrulloh [11] developed a technique to extract measurements from the automated segmentation of MH. In this technique, after the preprocessing step, the segmentation is processed by LGDF and Curvature-based Surface Cutting. A total of 30 images of $\mathrm{MH}$ cases were used and the average segmentation performance was of 19.84 seconds per image. The algorithm had an accuracy of $99.19 \%$, sensitivity of $85.18 \%$, Jaccard Index of $76.34 \%$ and DSC of $86.19 \%$. Keller [26] also created an algorithm to segment MH. The technique used was AMAL. It was necessary 10 different patients (24 images from each one). The mean, SD and the time to segment MH with the Dijkstra Shortest Path Search were of 0.043 $\mathrm{mm}, 0.013 \mathrm{~mm}$, and $0.039 \mathrm{~s}$, respectively.

Miri [96] proposed an algorithm to perform the automatic segmentation of the ILM boundary within optic nerve head $(\mathrm{ONH})$. The method identified the ILM surface using a Graph Theory and GVF methods. A total of 44 patients were used. The mean and SD were of $1.9 \pm 0.475 \mathrm{~mm}$ and the Pearson correlation was of $99.94 \%$. Zhang [5] proposed a technique similar to Miri [96] to segment CME for the retina with MH pathology. The 
coarse segmentation was made by AdaBoost classifier and the fine segmentation used Graph Cut algorithm. To evaluate the technique, 18 3D OCT volumes with MH and CME were used. The accuracy of TPR was of $84.5 \%$, FPR was of $1.8 \%$ and accuracy rate was of $98.6 \%$.

$\mathrm{Xu}$ [27] suggested a method for segmentation of 2D and 3D MH. The segmentation was performed using Multi-scale 3D Graph Search. A total of 51 eyes contained 128 B-scans were used. Patients with other diseases were excluded. The accuracy was of $96 \%$ and the mean error between the two experts was of 6.1\%. Different from Xu [27], Liu [19] developed a classification method of multiple macular pathologies, they were NM, ME, MH, and AMD. The segmentation approach was MSSP. A total of 326 macular SD-OCT scans were used. The algorithm achieved an AUC of 0.93 for all pathologies.

Sui [28] developed a choroidal segmentation algorithm. The techniques used were deep multi-scale CNN and Graph Search. A total of 23972 images were necessary. The mean and $\mathrm{SD}$ of absolute error in pixels were of $8.5 \pm 7.6$. Hu [65] sugested a segmentation technique of multiple retinal layer boundaries using the same techniques as Sui [28]. The method included 50 OCT images. The UE value in pixels for the mean, max, and SD were of 0.99, $1.13,0.10$, respectively.

Hussain [97] created an automatic method to segment the ILM and the Bruch's Membrane Opening (BMO). The techniques used were Canny Edge Detection, Dijkstra Shortest Path Search and Connected Component Analysis. A total of 18 SD-OCT volumes were used. The precision was of more than 95\%. The UE difference for BMO location and BMO-MRW were of $54.18 \pm 53.74 \mu \mathrm{m}$ and $58.62 \pm 43.12 \mu \mathrm{m}$ (mean \pm SD), respectively. Hussain [99] created an automatic method to identify 4 boundaries layers in the presence of 3 pathologies using the same techniques as Hussain [97]. A total of 3 datasets were used and 2 of them are public. The mean and SD of the root-mean-square error in pixels were of $1.57 \pm 0.69$.

Novosel [101] developed a segmentation method in 3D OCT volumes of retinal layers and focal lesions. The LCLS framework and Locally Adaptive Likelihood were applied. A total of 97 B-scans were used. The method achieved TPR of 93\% for fluid segmentation, DSC of $68 \%$ and MUE ranging from 4.9 to $8.3 \mu \mathrm{m}$ for drusen segmentation. Stankiewicz [106] sugested the segmentation of ERM from 3D volumes too. The used techniques were Peak Intensity Analysis and Graph Theory. A total of 141 B-scans were used. The results with Pixel Intensity Analysis in MSE and SD were 46.07 and $11.44 \mu \mathrm{m}$. The results with Graph Search technique in MSE and SD were 26.55 and $8.81 \mu \mathrm{m}$.

Naz [102] sugested the automatic segmentation of retinal layers using two techniques and compared using 108 OCT images: Kernel regression + GTDP (mean and SD of 0.004 $\pm 0.0001 \mu \mathrm{m}$ ) and Structure tensor (mean and SD of $0.289 \pm 0.0659 \mu \mathrm{m}$ ). Hassan [104] proposed an algorithm to detect fovea in OCT scans. The segmentation of six retina layers also used Structure Tensor and Canny Edge Detection. The dataset contains 120 OCT B-scans with three conditions: NM, ME and CSR. The overall accuracy was of $97.5 \%$.

Chiu [100] proposed the segmentation of three retinal boundaries from images with AMD, RPE and drusen. The segmentation were based on Dijkstra's Shortest Path Search, Graph Theory and DP. A total of 100 B-scans were used. The mean and SD were of 4.2 $\pm 2.8 \mu \mathrm{m}$. This work was one of the most compared and studied articles by researchers in this field. Tian [114] created an algorithm called OCTRIMA-3D that segments retinal layer boundaries of OCT volume. It is based in Dijkstra Shortest Path proposed by Chiu [100]. A total of 10 SD-OCT volume datasets were used. In addition, 100 SD-OCT images were necessary too. The MSE \pm SSE for the ILM surface were of $0.6 \pm 1.14$ pixels.

Athira [107] developed a technique to detect ME based on fractal texture analysis. Layers detection also used Dijkstra Shortest Path Search, and also Sparse Matrix and OTSU algorithm. A total of 100 normal and $100 \mathrm{ME}$ images were used. The algorithm had an accuracy of $97.5 \%$, sensitivity of $98.9 \%$ and specificity of $98.05 \%$. Dodo [109] created an algorithm to segment nine layers. Different from the others that used Dijkstra's Shortest Path 
in segmentation step, in this work it was applied to the preprocessing step. The initialisation used Fuzzy image processing. The segmentation was made by Level Set methods. A total of 200 images were used. The mean and SD in pixels were of $0.9643 \pm 0.014$.

Xiang [103] created a method to segment retinas with CSR. The technique used was RFC and Single Graph Live Wire Algorithm. A total of 48 images with 128 B-scans were used. The TPF, FPF and DSC in \% were of $92.73 \pm 15.03,0.05 \pm 0.09$ and $92.73 \pm$ 14.21, respectively. Lang [111] created a segmentation method for retinal layers in retinitis pigmentosa (RP). It also used RFC and Graph Search algorithm. A total of $512 \mathrm{~A}$-scans and B-scans varying from 19 to 49 for each patient were used. The boundary errors in $\mu \mathrm{m}$ over all subjects were specified in signed error and absolute error of - $0.9 \pm 2.65$ and $4.22 \pm$ 2.44 , and the layer thickness errors over all subjects were of $-0.03 \pm 3.33$ and $5.14 \pm 2.69$.

González-López [105] developed an automatic segmentation method for retinal boundary layers. The proposed technique was based on Snakes or Active Contours and it used 40 OCT images. The overall DSC was of $91.25 \%$ and the mean and SD for unsigned boundaries were of $1.27 \pm 1.06$ pixels. Rossant [113] sugested a segmentation method of layers for RP subjects using another approach of snakes, the PDS. The database included 95 images. Comparisons between the automatic segmentation to manual segmentation and comparisons of PDS model against Twin Snakes, Ribbon Snakes and Ribbon of Twins (ROT) were made. The PDS technique leaded to the best results with mean and SD of $1.3 \pm 0.33$ pixels.

Huang [80] proposed a classification method called ReLayNet for four conditions: NM, DME, drusen, and CNV. Two datasets were used. The first one contains 84484 OCT B-scans (averaged overall accuracy and SD were of 88.4 and 1.3). The second one contains a total of 8904 (averaged overall accuracy and SD were of 89.9 and 0.6). Nath [82] also proposed a segmentation and classification method to classify pathologies in normal or abnormal OCT images. The ROI was detected using attenuation coefficients by LCLS. There are no information about the database. The mean deviation for all interfaces were of 1.9 and $8.5 \mu \mathrm{m}$. Duan [110] created a technique to segment retinal layers using Region Growing method. Just as in Nath [82], nothing about the database was mencioned. The results shown were only qualitative. A visual comparison was made between the proposed method and other three techniques. It was possible conclude empirically that the proposed method had the best segmentation.

Niu [112] proposed a technique to segment retinal boundary layers using OTSU algorithm, Polynomial Fitting Function and Top-hat Filtering. A total of 3200 B-scans were necessary. The mean and SD in $\mu \mathrm{m}$ were of $0.22 \pm 0.24$. Hassan [81] developed an algorithm to classify macular conditions in NM, CSR and ME. Preprocessing and layer segmentation were necessary. For the segmentation step was also needed OTSU algorithm, also other methods, such as Tensor Grid and Canny Edge Detection. A total of 90 OCT volumes were necessary. The accuracy, sensitivity and specificity were of $97.78 \%, 96.77 \%$ and $100 \%$, respectively.

Gopinath [108] proposed an algorithm using deep learning, CNN and LSTM. Three datasets were used. For normal cases: dataset of Chiu (110 B-scans) and OCTRIMA3D (100 B-scans). For pathology cases: Chiu (220 B-scans). The mean and SD in pixels were of $1.78 \pm 0.78$ for Chiu-path, $1.16 \pm 0.46$ for Chiu-norm and $0.92 \pm 0.31$ for OCTRIMA3D. Hassan [1] also developed a deep learning classification technique for three retinal conditions: ME, CSR and NM. The segmentation step was performed based on Canny Edge Detection and OTSU algorithm. The dataset was composed by 90 time domain OCT (TD-OCT). The accuracy, sensitivity and specificity were of $97.77 \%, 100 \%$ and $93.33 \%$, respectively. Other work with similar approach was proposed by Fang [115]. It is a detection and recognition technique of multiple macular lesions: ERM, edema, and drusen in OCT images with Multi-instance Multilabel Learning. The segmentation of ROIs for different lesions was performed by SURF. A total of 823 clinically labeled OCT images were used. The results in \% were: accuracy of $88.72 \pm 0.84$, recall of $91.21 \pm 0.53$ and precision of $92.83 \pm 0.74$. 
El Tanboly [98] sugested the segmentation of the largest number of retinal layers, twelve, considering healthy and deseased subjects. The methods used were LCDG and MGRF. A total of 200 normal and diseased OCT scans were used. The accuracy using DSC, AC1 in $\%$, and $\mathrm{AD}$ in $\mu \mathrm{m}$ represented as mean and SD were of $0.763 \pm 0.1598,73.2 \pm 4.46$ and $6.87 \pm 2.78$. Besides segment twelve layers, Duan [43] also created an algorithm to detect 3D retinal boundary layers based on GDM. A total of 50 B-scans were used. The mean and SD of SE were of $-0.11 \pm 0.22 \mu \mathrm{m}$, absolute error (AE) were of $1.43 \pm 0.2 \mu \mathrm{m}$ and Hausdorff distance (HD) were of $7.3 \pm 0.67 \mu \mathrm{m}$. To compare and divide the articles in this review, some attributes were used. A total of 32 articles were utilized. The comparison is shown in Table 3.

Table 3. Comparison between the related works.

\begin{tabular}{|c|c|c|c|}
\hline Authors & Accuracy (\%) & Mean \pm SD $(\mathrm{mm})$ & Response time \\
\hline Nasrulloh et al. [11] & 99.19 & $0.9644 \pm 0.0015$ & 19.84 s/image \\
\hline Keller et al. [26] & NI & $0.043 \pm 0.013$ & $0.039 \mathrm{~s} / \mathrm{image}$ \\
\hline Miri et al. [96] & 99.94 & $1.9 \pm 0.475$ & NI \\
\hline Zhang et al. [5] & 98.6 & NI & NI \\
\hline Xu et al. [27] & 96 & $0.061 \pm 0.0$ & 38 s/image \\
\hline Liu et al. [19] & 93.1 & NI & NI \\
\hline Duan et al. [43] & NI & $0.0014 \pm 0.0002$ & 0.415 s/image \\
\hline Sui et al. [28] & NI & $8.5 \pm 7.6$ in pixel & 3.4 s/image \\
\hline Hu et al. [65] & NI & $0.99 \pm 0.1 \mathrm{in}$ pixel & 27.5 s/image \\
\hline Hussain et al. [97] & 97.27 & $0.059 \pm 0.0431$ & NI \\
\hline ElTanboly et al. [98] & NI & $0.0069 \pm 0.0029$ & NI \\
\hline Hussain et al. [99] & NI & $1.57 \pm 0.69$ in pixel & NI \\
\hline Chiu et al. [100] & NI & $0.0042 \pm 0.0028$ & $1.7 \mathrm{~s} /$ image \\
\hline Novosel et al. [101] & 89 & $0.0049 \sim 0.0083$ & NI \\
\hline Naz et al. [102] & NI & $0.004 \pm 0.0001$ in $\mu \mathrm{m}$ & NI \\
\hline Xiang et al. [103] & $92.73 \pm 14.21$ & NI & NI \\
\hline Hassan et al. [104] & 97.5 & NI & 5 s/image \\
\hline González-López et al. [105] & 91.25 & $1.27 \pm 1.06$ in pixel & NI \\
\hline Stankiewicz et al. [106] & NI & $0.0026 \pm 0.0088$ & NI \\
\hline Athira et al. [107] & 97.5 & NI & NI \\
\hline Gopinath et al. [108] & NI & $0.92 \pm 0.31$ in pixel & $4 \mathrm{~s} /$ volume \\
\hline Dodo et al. [109] & NI & $0.96 \pm 0.01$ in pixel & NI \\
\hline Duan et al. [110] & NI & NI & NI \\
\hline Lang et al. [111] & NI & $0.0042 \pm 0.0024$ & NI \\
\hline Niu et al. [112] & 99.96 & $0.0022 \pm 0.0024$ & NI \\
\hline Rossant et al. [113] & 87.4 & $1.3 \pm 0.33$ in pixel & NI \\
\hline Tian et al. [114] & NI & $0.6 \pm 0.0$ in pixel & $26.1 \mathrm{~s} /$ volume \\
\hline Huang et al. [80] & 89.9 & NI & NI \\
\hline Nath et al. [82] & NI & $0.0019 \pm 0.0085$ & NI \\
\hline Hassan and Hassan [81] & 97.78 & NI & NI \\
\hline Hassan et al. [1] & 97.77 & NI & 8 s/image \\
\hline Fang et al. [115] & 88.72 & NI & $0.25 \mathrm{~s} / \mathrm{image}$ \\
\hline
\end{tabular}

\section{Discussion}

The main objective of this review is approach the segmentation techniques of macular holes in OCT images, however the amount of works in this field is limited. Therefore, some works involving boundary layers segmentation in OCT images were used. Since the ILM layer is the most important layer for MH segmentation in OCT images, many of these works use 
techniques to segment it, but for other pathologies. A study of the selected works shows that many possibilities for segmentation of MH in OCT images are possible. Some approaches obtained accuracy upper 95\%, as examples: [11], [96], [5], [27], [99], [104], [107], [112], [81] and [1].

Some advantages can be mentioned in the works selected for this review. Liu [19] was the first study showing satisfactory results in automatic diagnosis in OCT images. It was important to serve as starting point to other works. Chiu [100] created one of the most compared and studied articles by researchers in this field. $\mathrm{Xu}$ [27] proposed a method that classify MH in four classes, this is an important step for the surgical aid. Keller [26] developed a method that can be applied to segment $\mathrm{MH}$ and other subjects in medical images. Zhang [5] created an algorithm that can segment CME excluding MH and blood vessels. The work of Miri [96] had smaller border errors than the comparative methods. Nasrulloh [11] developed a technique 61 times faster (2.46 minutes) than the original LGDF implementation. El Tanboly [98] had the first work segmenting twelve layers with all kinds of condition.

Duan [43] outperformed the Active Contour and Graph based approaches for segmenting retinal layers in both healthy and pathological images. Sui [28] developed a deep learning method which is unique in choroid segmentation. Hu [65] obtained more reliable probability maps using neural network. Novosel [101] developed an algorithm to segment layers and lesions. González-López [105] created an algorithm tolerant to noisy scenarios. Gopinath [108] developed a method that not requires any preprocessing step. Duan [110] successfully segmented the OCT image that contains some broken retinal layers. Tian [114] created an algorithm that not requires advanced denoising techniques.

Disadvantages can also be mentioned in the proposed works. In the preprocessing phase of Zhang [5], the delineation of MH was rough and contained failures. In Keller [26], the algorithm had data to find parameters like MH height and base width, but did not. In $\mathrm{Xu}$ [27], MH edge segmentation errors could be adjusted and patients with other diseases were excluded. Liu [19] found out that in their algorithm texture features may decrease effectiveness of the classifier. The work of Sui [28] spends about $10 \mathrm{~h}$ for each loop in training algorithm. Hussain [97] could not give a comparative analysis with other existing methods, because do not exist public database with segmentation of BMO or BMO-MRW.

Chiu [100] found a tradeoff between functionality and accuracy. Comparisons between the method of Novosel [101] and others was difficult, because there was no pattern of images and reference standard. González-López [105] sugested a model in which a completely fair comparison with the states-of-the-art seems extremely complicated because they used more than one dataset, each one with their own setting. Duan [110] created a method in which there is not quantitative analysis of the results and nothing about the database was mencioned. Tian [114] developed a methodology that sometimes has problem to segment the ILM boundary. The review showed that the latest segmentation techniques of MH in OCT images have not solution for all issues yet, and more research and improvement needs to be done.

\subsection{Future prospects}

Further researches are necessary to find out new approaches or improve the existing ones. A closer relationship between the medical community and the researchers strengthens the subject. Future prospects can allow the total use of MH segmentation systems in daily medical practices, they are:

- CV systems with a better accuracy and robustness to speckle noises;

- development of techniques that can automatically measure features of MH and classify them according with their classes;

- creation of a publicly database of OCT images with MH containing segment and feature information to help researchers on their works; 
- promoting a closer relationship between the involved parties in the system, such as engineers, physicians, technicians and government.

\section{Learned lessons}

Segmentation techniques are fundamental to aid in the diagnosis of medical pathologies. Researchers are constantly looking for the best segmentation techniques. The advancement of computing is allowing practices with increasing levels of accuracy. Image preprocessing is almost always required before applying some segmentation technique, but some deep learning tools already do not require image preprocessing. Despite the growth of deep learning techniques, new preprocessing and segmentation techniques are constantly appearing.

There are some softwares that can be used for CV tasks, but the predominance is the Matlab and Python as integrated development environment. There are numerous public and private databases for macular pathologies, but for macular holes there is still no well-defined database with manual segmentation for comparison porpuse, per example. The number of experts working on the ground truth of articles can range from one to five. Comparisons for validation work may use one to eight state-of-the-art. Advantages and disadvantages can be observed in each work, depending on the technique used. A perfect result for segmentation of $\mathrm{MH}$ or similar pathology has not been found yet. Validation metrics can vary, this will depend on what kind of parameter you want to focus on.

\section{Conclusion}

This paper elaborated a review of CV techniques applied to segmentation of macular holes and similar techniques that can be applied on this pathology. The work used papers published from the year 2011 to nowadays on Science Direct, IEEE, PubMed and Google scholar bases, which makes a recent review. Progress has been performed in this field. However, though the area of diagnostics requires more studies. The issue is important to the scientific community, due the need of boundary segmentation in other tasks too.

In general, the revised papers show potential for the joining of $\mathrm{CV}$ technology with medical diagnostics. Many challenges still need to be overcome for their full admission in medical practices. For this, a good partnership between researchers and physicians is needed. The total acceptance to use this technology in medical practices will only work by joining forces between health professionals, researchers, government, engineers and partners. Thus, with these efforts will be possible to spread this technology and make it operational. The reduction of errors is a requirement for the acceptable use of these technologies.

The best results could be analyzed due to well done research, considering the best keywords, in well-known scientific bases. This work may be a source of study for researchers wishing to delve into MH segmentation techniques. This review may serve as a basis for studies and developments of techniques even more robust than those mentioned here, with the aim of solving the problems that still exist in automatic MH segmentation.

\section{Acknowledgements}

The author thanks IFCE (Instituto Federal do Ceará), Prof. Dr. Auzuir Ripardo de Alexandria and Escola Cearense de Oftalmologia for the academic support and FUNCAP (Fundação Cearense de Apoio ao Desenvolvimento Científico e Tecnológico) for the financial support.

\section{Conflicts of Interest}

There is no conflict of interest. 


\section{References}

[1] Hassan, Bilal, Gulistan Raja, Taimur Hassan, and M. Usman Akram. "Structure tensor based automated detection of macular edema and central serous retinopathy using optical coherence tomography images." JOSA A 33, no. 4 (2016): 455-463

[2] Slokom, N., H. Trabelsi, and I. Zghal. "Segmentation of cyctoids macular edema in optical cohenrence tomography." In 2016 2nd International Conference on Advanced Technologies for Signal and Image Processing (ATSIP), pp. 303-306. 2016.

[3] Hood, Donald C., Susan C. Anderson, Michael Wall, and Randy H. Kardon. "Structure versus function in glaucoma: an application of a linear model." Investigative ophthalmology \& visual science 48, no. 8 (2007): 3662-3668.

[4] Van Dijk, Hille W., Frank D. Verbraak, Pauline HB Kok, Mona K. Garvin, Milan Sonka, Kyungmoo Lee, J. Hans DeVries et al. 'Decreased retinal ganglion cell layer thickness in patients with type 1 diabetes." Investigative ophthalmology \& visual science 51, no. 7 (2010): 3660-3665.

[5] Zhang, Li, Weifang Zhu, Fei Shi, Haoyu Chen, and Xinjian Chen. "Automated segmentation of intraretinal cystoid macular edema for retinal 3D OCT images with macular hole.” In 2015 IEEE 12th International Symposium on Biomedical Imaging (ISBI), pp. 1494-1497. IEEE, 2015.

[6] Faghihi, Hooshang, Fariba Ghassemi, Khalil Ghasemi Falavarjani, Ghasem Saeedi Anari, Mona Safizadeh, and Kourosh Shahraki. "Spontaneous closure of traumatic macular holes." Canadian Journal of Ophthalmology/Journal Canadien d'Ophtalmologie 49, no. 4 (2014): 395-398.

[7] Thapa, Suman S., Raba Thapa, Indira Paudyal, Shankar Khanal, Jaskirat Aujla, Govinda Paudyal, and Ger van Rens. "Prevalence and pattern of vitreo-retinal diseases in Nepal: the Bhaktapur glaucoma study." BMC ophthalmology 13, no. 1 (2013): 9.

[8] Luckie, Alan, and Wilson Heriot. "Macular holes: pathogenesis, natural history and surgical outcomes." Australian and New Zealand journal of ophthalmology 23, no. 2 (1995): 93-100.

[9] Klein, Ronald, Barbara EK Klein, and Kathryn LP Linton. "Prevalence of age-related maculopathy: the Beaver Dam Eye Study." Ophthalmology 99, no. 6 (1992): 933-943.

[10] Velez-Montoya, Raul, Scott CN Oliver, Jeffrey L. Olson, Stuart L. Fine, Hugo QuirozMercado, and Naresh Mandava. "Current knowledge and trends in age-related macular degeneration: genetics, epidemiology, and prevention." Retina 34, no. 3 (2014): 423441 .

[11] Nasrulloh, Amar V., Chris G. Willcocks, Philip TG Jackson, Caspar Geenen, Maged S. Habib, David HW Steel, and Boguslaw Obara. "Multi-Scale Segmentation and Surface Fitting for Measuring 3-D Macular Holes." IEEE transactions on medical imaging 37, no. 2 (2017): 580-589.

[12] Chhablani, Jay, Mitali Khodani, Abdullah Hussein, Sailaja Bondalapati, Harsha B. Rao, Raja Narayanan, and Aditya Sudhalkar. "Role of macular hole angle in macular hole closure." British Journal of Ophthalmology 99, no. 12 (2015): 1634-1638.

[13] Madi, Haifa A., Ibrahim Masri, and David H. Steel. "Optimal management of idiopathic macular holes." Clinical ophthalmology (Auckland, NZ) 10 (2016): 97.

[14] Steel, D. H. W., C. Parkes, V. T. Papastavrou, P. J. Avery, I. A. El-Ghrably, M. S. Habib, M. T. Sandinha et al. "Predicting macular hole closure with ocriplasmin based on spectral domain optical coherence tomography." Eye 30, no. 5 (2016): 740.

[15] Philippakis, E., M. Legrand, M. El Sanharawi, A. Erginay, A. Couturier, and R. Tadayoni. "Measurement of full-thickness macular hole size using en face optical coherence tomography." Eye 32, no. 3 (2018): 590.

[16] Fabritius, Tapio, Shuichi Makita, Masahiro Miura, Risto Myllylä, and Yoshiaki Yasuno. "Automated segmentation of the macula by optical coherence tomography." Optics 
express 17, no. 18 (2009): 15659-15669.

[17] de Moura, Joaquim, Jorge Novo, Susana Penas, Marcos Ortega, Jorge Silva, and Ana Maria Mendonça. "Automatic Characterization of the Serous Retinal Detachment Associated with the Subretinal Fluid Presence in Optical Coherence Tomography Images." Procedia Computer Science 126 (2018): 244-253.

[18] Altaweel, Michael, and Michael Ip. "Macular hole: improved understanding of pathogenesis, staging, and management based on optical coherence tomography." In Seminars in ophthalmology, vol. 18, no. 2, pp. 58-66. Taylor \& Francis, 2003.

[19] Liu, Yu-Ying, Mei Chen, Hiroshi Ishikawa, Gadi Wollstein, Joel S. Schuman, and James M. Rehg. "Automated macular pathology diagnosis in retinal OCT images using multi-scale spatial pyramid and local binary patterns in texture and shape encoding." Medical image analysis 15, no. 5 (2011): 748-759.

[20] Ip MS, Baker BJ, Duker JS et al (2002) Anatomical outcomes of surgery for idiopathic macular hole as determined by optical coherence tomography. Arch Ophthalmol 120:29-35.

[21] Tadayoni R, Svorenova I, Erginay A, Gaudric A, Massin P. Decreased retinal sensitivity after internal limiting membrane peeling for macular hole surgery. Br J Ophthalmol 2012; 96(12): 1513-1516.

[22] Haller JA, Stalmans P, Benz MS, Gandorfer A, Pakola SJ, Girach A et al. Efficacy of intravitreal ocriplasmin for treatment of vitreomacular adhesion: subgroup analyses from two randomized trials. Ophthalmology 2015; 122(1): 117-122.

[23] Freeman, William R., Stanley P. Azen, Jung W. Kim, Wael El-Haig, Daniel R. Mishell, and Ian Bailey. "Vitrectomy for the treatment of full-thickness stage 3 or 4 macular holes: results of a multicentered randomized clinical trial." Archives of Ophthalmology 115, no. 1 (1997): 11-21.

[24] Park, Donald W., Jack O. Sipperley, Scott R. Sneed, Pravin U. Dugel, and Jaclin Jacobsen. "Macular hole surgery with internal-limiting membrane peeling and intravitreous air." Ophthalmology 106, no. 7 (1999): 1392-1398.

[25] Haritoglou, Christos, Carolin A. Gass, Markus Schaumberger, Oliver Ehrt, Arnd Gandorfer, and Anselm Kampik. "Macular changes after peeling of the internal limiting membrane in macular hole surgery." American journal of ophthalmology 132, no. 3 (2001): 363-368.

[26] Keller, Brenton, David Cunefare, Dilraj S. Grewal, Tamer H. Mahmoud, Joseph A. Izatt, and Sina Farsiu. "Length-adaptive graph search for automatic segmentation of pathological features in optical coherence tomography images." Journal of biomedical optics 21, no. 7 (2016): 076015.

[27] Xu, David, Alex Yuan, Peter K. Kaiser, Sunil K. Srivastava, Rishi P. Singh, Jonathan E. Sears, Daniel F. Martin, and Justis P. Ehlers. ”A novel segmentation algorithm for volumetric analysis of macular hole boundaries identified with optical coherence tomography." Investigative ophthalmology \& visual science 54, no. 1 (2013): 163-169.

[28] Sui, Xiaodan, Yuanjie Zheng, Benzheng Wei, Hongsheng Bi, Jianfeng Wu, Xuemei Pan, Yilong Yin, and Shaoting Zhang. "Choroid segmentation from optical coherence tomography with graph-edge weights learned from deep convolutional neural networks." Neurocomputing 237 (2017): 332-341.

[29] Gong, Guanghua, Hongming Zhang, and Minyu Yao. "Speckle noise reduction algorithm with total variation regularization in optical coherence tomography." Optics express 23, no. 19 (2015): 24699-24712.

[30] Tang, Chang, Xiao Zheng, and Lijuan Cao. "OCT despeckling via weighted nuclear norm constrained non-local low-rank representation." Laser Physics Letters 14, no. 10 (2017): 106001.

[31] Huang, David, Eric A. Swanson, Charles P. Lin, Joel S. Schuman, William G. Stinson, Warren Chang, Michael R. Hee, Thomas Flotte, Kenton Gregory, and Carmen A. 
Puliafito. "Optical coherence tomography." science 254, no. 5035 (1991): 1178-1181.

[32] Coscas, Gabriel, José Cunha-Vaz, and Gisèle Soubrane. "Macular edema: definition and basic concepts." In Macular Edema, vol. 47, pp. 1-9. Karger Publishers, 2010.

[33] Tranos, Paris G., Sanjeewa S. Wickremasinghe, Nikos T. Stangos, Fotis Topouzis, Ioannis Tsinopoulos, and Carlos E. Pavesio. "Macular edema." Survey of ophthalmology 49, no. 5 (2004): 470-490.

[34] Schuman, Joel S., Tamar Pedut-Kloizman, Ellen Hertzmark, Michael R. Hee, Jason R. Wilkins, Jeffery G. Coker, Carmen A. Puliafito, James G. Fujimoto, and Eric A. Swanson. "Reproducibility of nerve fiber layer thickness measurements using optical coherence tomography." Ophthalmology 103, no. 11 (1996): 1889-1898.

[35] Schuman, Joel S. Optical coherence tomography of ocular diseases. Edited by Carmen A. Puliafito, James G. Fujimoto, and Jay S. Duker. New Jersey:: Slack, 2004.

[36] Drexler, Wolfgang, Harald Sattmann, Boris Hermann, Tony H. Ko, Michael Stur, Angelika Unterhuber, Christoph Scholda et al. "Enhanced visualization of macular pathology with the use of ultrahigh-resolution optical coherence tomography." Archives of ophthalmology 121, no. 5 (2003): 695-706.

[37] Ricco, Susanna, Mei Chen, Hiroshi Ishikawa, Gadi Wollstein, and Joel Schuman. "Correcting motion artifacts in retinal spectral domain optical coherence tomography via image registration." In International Conference on Medical Image Computing and Computer-Assisted Intervention, pp. 100-107. Springer, Berlin, Heidelberg, 2009.

[38] Xu, Juan, Hiroshi Ishikawa, Gadi Wollstein, and Joel S. Schuman. "3D OCT eye movement correction based on particle filtering." In 2010 Annual International Conference of the IEEE Engineering in Medicine and Biology, pp. 53-56. IEEE, 2010.

[39] Hu, Junjie, Yuanyuan Chen, and Zhang Yi. "Automated segmentation of macular edema in OCT using deep neural networks." Medical image analysis 55 (2019): 216227.

[40] Schmitt, Joseph M., S. H. Xiang, and Kin Man Yung. "Speckle in optical coherence tomography." Journal of biomedical optics 4, no. 1 (1999): 95-106.

[41] Grzywacz, Norberto Mauricio, Joaquín De Juan, Claudia Ferrone, Daniela Giannini, David Huang, Giorgio Koch, Valentina Russo, Ou Tan, and Carlo Bruni. "Statistics of optical coherence tomography data from human retina." IEEE transactions on medical imaging 29, no. 6 (2010): 1224-1237.

[42] Lindenmaier, Andras A., Leigh Conroy, Golnaz Farhat, Ralph S. DaCosta, Costel Flueraru, and I. Alex Vitkin. "Texture analysis of optical coherence tomography speckle for characterizing biological tissues in vivo." Optics letters 38, no. 8 (2013): $1280-1282$.

[43] Duan, Jinming, Christopher Tench, Irene Gottlob, Frank Proudlock, and Li Bai. "Automated segmentation of retinal layers from optical coherence tomography images using geodesic distance." Pattern Recognition 72 (2017): 158-175.

[44] Chen, Huaiguang, Shujun Fu, Hong Wang, Yuliang Li, and Fengling Wang. "Speckle reduction based on fractional-order filtering and boosted singular value shrinkage for optical coherence tomography image." Biomedical Signal Processing and Control 52 (2019): 281-292.

[45] Lv, Hongli, Shujun Fu, Caiming Zhang, and Lin Zhai. "Speckle noise reduction for optical coherence tomography based on adaptive 2D dictionary." Laser Physics Letters 15, no. 5 (2018): 055401.

[46] Sadda SR, Wu Z, Walsh AC, et al. Errors in retinal thickness measurements obtained by optical coherence tomography. Ophthalmology 2006;113:285-93.

[47] Krebs I, Haas P, Zeiler F, et al. Optical coherence tomography: limits of the retinal-mapping program in age-related macular degeneration. Br J Ophthalmol 2008;92:933-5. 
[48] Hughes, Michael, Marika Spring, and Adrian Podoleanu. "Speckle noise reduction in optical coherence tomography of paint layers." Applied optics 49, no. 1 (2010): 99-107.

[49] Desjardins, A. E., B. J. Vakoc, G. J. Tearney, and B. E. Bouma. "Speckle reduction in OCT using massively-parallel detection and frequency-domain ranging." Optics Express 14, no. 11 (2006): 4736-4745.

[50] Iftimia, Nicusor, Brett E. Bouma, and Guillermo J. Tearney. "Speckle reduction in optical coherence tomography by" path length encoded" angular compounding." Journal of biomedical optics 8, no. 2 (2003): 260-263.

[51] Jørgensen, Thomas Martini, Jakob Thomadsen, Ulrik Christensen, Wael Soliman, and Birgit A. Sander. "Enhancing the signal-to-noise ratio in ophthalmic optical coherence tomography by image registration-method and clinical examples." Journal of biomedical optics 12, no. 4 (2007): 041208.

[52] Szkulmowski, Maciej, Iwona Gorczynska, Daniel Szlag, Marcin Sylwestrzak, Andrzej Kowalczyk, and Maciej Wojtkowski. "Efficient reduction of speckle noise in Optical Coherence Tomography." Optics express 20, no. 2 (2012): 1337-1359.

[53] Waldstein, Sebastian M., Bianca S. Gerendas, Alessio Montuoro, Christian Simader, and Ursula Schmidt-Erfurth. "Quantitative comparison of macular segmentation performance using identical retinal regions across multiple spectral-domain optical coherence tomography instruments." British Journal of Ophthalmology 99, no. 6 (2015): 794-800.

[54] Xu, Min, Chen Tang, Mingming Chen, Yue Qiu, and Zhenkun Lei. "Texture preservation and speckle reduction in optical coherence tomography using the shearletbased total variation algorithm." Optics and Lasers in Engineering 122 (2019): 265283.

[55] VSRI. Vision Science and Advanced Retinal Imaging Laboratory. University of California, Davis, 2019. URL http://vsri.ucdavis.edu/research/retinal/ oct.

[56] Takahashi, Atsushi, Taiji Nagaoka, and Akitoshi Yoshida. "Enhanced vitreous imaging optical coherence tomography in primary macular holes." International ophthalmology 36, no. 3 (2016): 355-363.

[57] Gupta, Vishali, Amod Gupta, and Mangat R. Dogra. Atlas of optical coherence tomography of macular diseases. CRC Press, 2004.

[58] Kong, Mingui, Da Ye Choi, Gyule Han, Yun-Mi Song, Sung Yong Park, Joohon Sung, Sungsoon Hwang, and Don-Il Ham. "Measurable Range of Subfoveal Choroidal Thickness With Conventional Spectral Domain Optical Coherence Tomography." Translational vision science \& technology 7, no. 5 (2018): 16-16.

[59] Lu, Donghuan, Morgan Heisler, Sieun Lee, Gavin Ding, Marinko V. Sarunic, and Mirza Faisal Beg. "Retinal fluid segmentation and detection in optical coherence tomography images using fully convolutional neural network." arXiv preprint arXiv:1710.04778 (2017).

[60] Joussen, Antonia M., Thomas W. Gardner, Bernd Kirchhof, and Stephen J. Ryan, eds. Retinal vascular disease. Vol. 75. Heidelberg: Springer, 2007.

[61] Potsaid, Benjamin, Iwona Gorczynska, Vivek J. Srinivasan, Yueli Chen, James Jiang, Alex Cable, and James G. Fujimoto. "Ultrahigh speed spectral/Fourier domain OCT ophthalmic imaging at 70,000 to 312,500 axial scans per second." Optics express 16, no. 19 (2008): 15149-15169.

[62] Wojtkowski, Maciej. "High-speed optical coherence tomography: basics and applications." Applied optics 49, no. 16 (2010): D30-D61.

[63] Zawadzki, Robert J., Barry Cense, Yan Zhang, Stacey S. Choi, Donald T. Miller, and John S. Werner. "Ultrahigh-resolution optical coherence tomography with monochromatic and chromatic aberration correction." Optics Express 16, no. 11 (2008): 
8126-8143.

[64] Salarian, Mahdi, Rashid Ansari, Justin Wanek, and Mahnaz Shahidi. "Acuurate segmentation of retina nerve fiber layer in OCT images." In 2015 IEEE International Conference on Electro/Information Technology (EIT), pp. 653-656. IEEE, 2015.

[65] Hu, Kai, Binwei Shen, Yuan Zhang, Chunhong Cao, Fen Xiao, and Xieping Gao. "Automatic segmentation of retinal layer boundaries in OCT images using multiscale convolutional neural network and graph search.” Neurocomputing 365 (2019): 302313.

[66] Girish, G. N., Abhishek R. Kothari, and Jeny Rajan. "Automated segmentation of intra-retinal cysts from optical coherence tomography scans using marker controlled watershed transform." In 2016 38th Annual International Conference of the IEEE Engineering in Medicine and Biology Society (EMBC), pp. 1292-1295. IEEE, 2016.

[67] Wang, Chuang, Ya Xing Wang, and Yongmin Li. "Automatic choroidal layer segmentation using markov random field and level set method." IEEE journal of biomedical and health informatics 21, no. 6 (2017): 1694-1702.

[68] Fernández, Delia Cabrera, Harry M. Salinas, and Carmen A. Puliafito. "Automated detection of retinal layer structures on optical coherence tomography images." Optics Express 13, no. 25 (2005): 10200-10216.

[69] Vermeer, K. A., J. Van der Schoot, H. G. Lemij, and J. F. De Boer. "Automated segmentation by pixel classification of retinal layers in ophthalmic OCT images." Biomedical optics express 2, no. 6 (2011): 1743-1756.

[70] George, Neetha, and C. V. Jiji. "Two stage contour evolution for automatic segmentation of choroid and cornea in OCT images." Biocybernetics and Biomedical Engineering (2019).

[71] Ho J, Sull AC, Vuong LN, et al. Assessment of artifacts and reproducibility across spectral- and time-domain optical coherence tomography devices. Ophthalmology 2009;116:1960-70.

[72] Mylonas G, Ahlers C, Malamos P, et al. Comparison of retinal thickness measurements and segmentation performance of four different spectral and time domain OCT devices in neovascular age-related macular degeneration. Br J Ophthalmol 2009;93:1453-60.

[73] Berg, M. "IT-supported skill-mix change and standardisation in integrated eyecare: lessons from two screening projects in The Netherlands." International journal of integrated care 7 (2007): e15-e15.

[74] Marrugo, Andrés G., María S. Millán, Gabriel Cristóbal, Salvador Gabarda, Michal Sorel, and Filip Sroubek. "Image analysis in modern ophthalmology: from acquisition to computer assisted diagnosis and telemedicine." In Optics, Photonics, and Digital Technologies for Multimedia Applications II, vol. 8436, p. 84360C. International Society for Optics and Photonics, 2012.

[75] Vercellin, Alice C. Verticchio, Firas Jassim, Linda Yi-Chieh Poon, Edem Tsikata, Boy Braaf, Sneha Shah, Geulah Ben-David et al. "Diagnostic capability of threedimensional macular parameters for glaucoma using optical coherence tomography volume scans." Investigative ophthalmology \& visual science 59, no. 12 (2018): 49985010.

[76] Montuoro, Alessio, Sebastian M. Waldstein, Bianca S. Gerendas, Ursula SchmidtErfurth, and Hrvoje Bogunović. "Joint retinal layer and fluid segmentation in OCT scans of eyes with severe macular edema using unsupervised representation and autocontext." Biomedical optics express 8, no. 3 (2017): 1874-1888.

[77] Petrick, Nicholas, Berkman Sahiner, Samuel G. Armato III, Alberto Bert, Loredana Correale, Silvia Delsanto, Matthew T. Freedman et al. "Evaluation of computer-aided detection and diagnosis systems a." Medical physics 40, no. 8 (2013): 087001.

[78] Ramzan, Aneeqa, Muhammad Usman Akram, Arslan Shaukat, Sajid Gul Khawaja, Ubaid Ullah Yasin, and Wasi Haider Butt. "Automated glaucoma detection using 
retinal layers segmentation and optic cup-to-disc ratio in optical coherence tomography images." IET Image Processing 13, no. 3 (2018): 409-420.

[79] Gerendas, Bianca S., Julia-Sophie Kroisamer, Wolf Buehl, Sandra M. Rezar-Dreindl, Katharina M. Eibenberger, Eleonore Pablik, Ursula Schmidt-Erfurth, and Stefan Sacu. "Correlation between morphological characteristics in spectral-domain-optical coherence tomography, different functional tests and a patient's subjective handicap in acute central serous chorioretinopathy." Acta ophthalmologica 96, no. 7 (2018): e776-e782.

[80] Huang, Laifeng, Xingxin He, Leyuan Fang, Hossein Rabbani, and Xiangdong Chen. "Automatic Classification of Retinal Optical Coherence Tomography Images With Layer Guided Convolutional Neural Network." IEEE Signal Processing Letters 26, no. 7 (2019): 1026-1030.

[81] Hassan, Bilal, and Taimur Hassan. "Fully automated detection, grading and 3D modeling of maculopathy from OCT volumes." In 2019 2nd International Conference on Communication, Computing and Digital systems (C-CODE), pp. 252-257. IEEE, 2019.

[82] Nath, Sravani S., B. K. Anoop, and Perumal Sankar. "Classification of Outer Retinal Layers Based on KNN-Classifier." In 2018 International Conference on Emerging Trends and Innovations In Engineering And Technological Research (ICETIETR), pp. 1-4. IEEE, 2018.

[83] Valente, Igor Rafael S., Paulo César Cortez, Edson Cavalcanti Neto, José Marques Soares, Victor Hugo C. de Albuquerque, and João Manuel RS Tavares. "Automatic 3D pulmonary nodule detection in CT images: a survey." Computer methods and programs in biomedicine 124 (2016): 91-107.

[84] Ayache, Nicholas. "Medical computer vision, virtual reality and robotics." Image and Vision Computing 13, no. 4 (1995): 295-313.

[85] Rosenfeld, Azriel. "Computer vision: basic principles." Proceedings of the IEEE 76, no. 8 (1988): 863-868.

[86] Mendes, Odilon Linhares Carvalho; Borille, Giovanna Miceli Ronzani. "Computer vision systems in unmanned aerial vehicle: a review." Journal of Mechatronics Engineering 2, no. 2 (2019): 11-22.

[87] Andreopoulos, Alexander, and John K. Tsotsos. "50 years of object recognition: Directions forward." Computer vision and image understanding 117, no. 8 (2013): 827-891.

[88] Alves de Souza, Thamiris, Caterine Silva de Oliveira, Cesar Sanin, and Edward Szczerbicki. "From knowledge based vision systems to cognitive vision systems: a review." Procedia Computer Science 126 (2018): 1855-1864.

[89] Bellotti, Roberto, Francesco De Carlo, Sonia Tangaro, Gianfranco Gargano, Giuseppe Maggipinto, Marcello Castellano, Raffaella Massafra et al. "A completely automated CAD system for mass detection in a large mammographic database." Medical physics 33, no. 8 (2006): 3066-3075.

[90] Duke, Duke dataset, 2013. URL http://people.duke.edu/ sf59/RPEDC_ Ophth_2013_dataset.htm.

[91] OCTRIMA-3D, OCT Retinal IMage Analysis 3D, 2015. URL https://journals . plos.org/plosone/article?id=10.1371/journal . pone. 0133908.

[92] Mendeley, Labeled Optical Coherence Tomography (OCT) and Chest X-Ray Images for Classification, 2018. URL https://data.mendeley.com/datasets / rscbjbr9sj/2.

[93] Reza Rasti, Noor Eye Hospital in Tehran, 2018. URL https://sites .google.com/ site/hosseinrabbanikhorasgani/datasets-1.

[94] Reza Rasti, Hossein Rabbani, Alireza Mehri, Fedra Hajizadeh, "Macular OCT Classification using a Multi-Scale Convolutional Neural Network Ensemble”, IEEE 
Transactions on Medical Imaging, vol. 37, no. 4, pp. 1024-1034, April 2018.

[95] OCTID, Optical Coherence Tomography Image Database, 2018. URL https: //dataverse. scholarsportal. info/dataset. xhtml?persistentId=doi: 10.5683/SP/MBMQGD.

[96] Miri, Mohammad Saleh, Victor A. Robles, Michael D. Abràmoff, Young H. Kwon, and Mona K. Garvin. 'Incorporation of gradient vector flow field in a multimodal graphtheoretic approach for segmenting the internal limiting membrane from glaucomatous optic nerve head-centered SD-OCT volumes." Computerized Medical Imaging and Graphics 55 (2017): 87-94.

[97] Hussain, Md Akter, Alauddin Bhuiyan, and Kotagiri Ramamohanarao. "Disc segmentation and BMO-MRW measurement from SD-OCT image using graph search and tracing of three bench mark reference layers of retina." In 2015 IEEE International Conference on Image Processing (ICIP), pp. 4087-4091. IEEE, 2015.

[98] El Tanboly, Ahmed, Marwa Ismail, A. Switala, M. Mahmoud, Ahmed Soliman, T. Neyer, A. Palacio et al. "A novel automatic segmentation of healthy and diseased retinal layers from OCT scans." In 2016 IEEE International Conference on Image Processing (ICIP), pp. 116-120. IEEE, 2016.

[99] Hussain, Md Akter, Alauddin Bhuiyan, Andrew Turpin, Chi D. Luu, R. Theodore Smith, Robyn H. Guymer, and Ramamohanrao Kotagiri. "Automatic identification of pathology-distorted retinal layer boundaries using SD-OCT imaging." IEEE Transactions on Biomedical Engineering 64, no. 7 (2016): 1638-1649.

[100] Chiu, Stephanie J., Joseph A. Izatt, Rachelle V. O'Connell, Katrina P. Winter, Cynthia A. Toth, and Sina Farsiu. "Validated automatic segmentation of AMD pathology including drusen and geographic atrophy in SD-OCT images." Investigative ophthalmology \& visual science 53, no. 1 (2012): 53-61.

[101] Novosel, Jelena, Koenraad A. Vermeer, Jan H. de Jong, Ziyuan Wang, and Lucas J. van Vliet. "Joint segmentation of retinal layers and focal lesions in 3-D OCT data of topologically disrupted retinas." IEEE transactions on medical imaging 36, no. 6 (2017): 1276-1286.

[102] Naz, Samra, M. Usman Akram, and Shoab A. Khan. "Automated segmentation of retinal layers from OCT images using structure tensor and kernel regression+ GTDP approach.” In 2017 1st International Conference on Next Generation Computing Applications (NextComp), pp. 98-102. IEEE, 2017.

[103] Xiang, Dehui, Geng Chen, Fei Shi, Weifang Zhu, Qinghuai Liu, Songtao Yuan, and Xinjian Chen. "Automatic retinal layer segmentation of OCT images with central serous retinopathy." IEEE journal of biomedical and health informatics 23, no. 1 (2018): 283-295.

[104] Hassan, Bilal, Ramsha Ahmed, and Bo Li. "Automated Foveal Detection in OCT Scans." In 2018 IEEE International Symposium on Signal Processing and Information Technology (ISSPIT), pp. 419-422. IEEE, 2018.

[105] González-López, A., J. de Moura, J. Novo, M. Ortega, and M. G. Penedo. "Robust segmentation of retinal layers in optical coherence tomography images based on a multistage active contour model." Heliyon 5, no. 2 (2019): e01271.

[106] Stankiewicz, Agnieszka, Tomasz Marciniak, Adam Dabrowski, Marcin Stopa, Piotr Rakowicz, and Elzbieta Marciniak. "Novel full-automatic approach for segmentation of epiretinal membrane from 3D OCT images." In 2017 Signal Processing: Algorithms, Architectures, Arrangements, and Applications (SPA), pp. 100-105. IEEE, 2017.

[107] Athira, S. C., Reena M. Roy, and R. P. Aneesh. "Computerized Detection of Macular Edema Using OCT Images Based on Fractal Texture Analysis.” In 2018 International CET Conference on Control, Communication, and Computing (IC4), pp. 326-330. IEEE, 2018.

[108] Gopinath, Karthik, Samrudhdhi B. Rangrej, and Jayanthi Sivaswamy. ”A deep 
learning framework for segmentation of retinal layers from OCT images." In 2017 th IAPR Asian Conference on Pattern Recognition (ACPR), pp. 888-893. IEEE, 2017.

[109] Dodo, Bashir I., Yongmin Li, Allan Tucker, Djibril Kaba, and Xiaohui Liu. "Retinal OCT Segmentation Using Fuzzy Region Competition and Level Set Methods." In 2019 IEEE 32nd International Symposium on Computer-Based Medical Systems (CBMS), pp. 93-98. IEEE, 2019.

[110] Duan, Jinming, Christopher Tench, Irene Gottlob, Frank Proudlock, and Li Bai. "Optical coherence tomography image segmentation." In 2015 IEEE International Conference on Image Processing (ICIP), pp. 4278-4282. IEEE, 2015.

[111] Lang, Andrew, Aaron Carass, Ava K. Bittner, Howard S. Ying, and Jerry L. Prince. "Improving graph-based OCT segmentation for severe pathology in Retinitis Pigmentosa patients." In Medical Imaging 2017: Biomedical Applications in Molecular, Structural, and Functional Imaging, vol. 10137, p. 101371M. International Society for Optics and Photonics, 2017.

[112] Niu, Sijie, Qiang Chen, Luis de Sisternes, Daniel L. Rubin, Weiwei Zhang, and Qinghuai Liu. "Automated retinal layers segmentation in SD-OCT images using dualgradient and spatial correlation smoothness constraint." Computers in biology and medicine 54 (2014): 116-128.

[113] Rossant, Florence, Isabelle Bloch, Itebeddine Ghorbel, and Michel Paques. "Parallel Double Snakes. Application to the segmentation of retinal layers in 2D-OCT for pathological subjects." Pattern Recognition 48, no. 12 (2015): 3857-3870.

[114] Tian, Jing, Boglárka Varga, Gábor Márk Somfai, Wen-Hsiang Lee, William E. Smiddy, and Delia Cabrera DeBuc. "Real-time automatic segmentation of optical coherence tomography volume data of the macular region." PloS one 10, no. 8 (2015): e0133908.

[115] Fang, Leyuan, Liumao Yang, Shutao Li, Hossein Rabbani, Zhimin Liu, Qinghua Peng, and Xiangdong Chen. "Automatic detection and recognition of multiple macular lesions in retinal optical coherence tomography images with multi-instance multilabel learning." Journal of biomedical optics 22, no. 6 (2017): 066014.

[116] Qian, Pengjiang, Kaifa Zhao, Yizhang Jiang, Kuan-Hao Su, Zhaohong Deng, Shitong Wang, and Raymond F. Muzic Jr. "Knowledge-leveraged transfer fuzzy C-Means for texture image segmentation with self-adaptive cluster prototype matching." Knowledgebased systems 130 (2017): 33-50.

[117] Roy, Abhijit Guha, Sailesh Conjeti, Sri Phani Krishna Karri, Debdoot Sheet, Amin Katouzian, Christian Wachinger, and Nassir Navab. "ReLayNet: retinal layer and fluid segmentation of macular optical coherence tomography using fully convolutional networks." Biomedical optics express 8, no. 8 (2017): 3627-3642.

[118] Xu, Chenyang, and Jerry L. Prince. "Snakes, shapes, and gradient vector flow." IEEE Transactions on image processing 7, no. 3 (1998): 359-369.

[119] Oguz, Ipek, and Milan Sonka. "LOGISMOS-B: layered optimal graph image segmentation of multiple objects and surfaces for the brain." IEEE transactions on medical imaging 33, no. 6 (2014): 1220-1235.

[120] Lee, Cecilia S., Ariel J. Tyring, Nicolaas P. Deruyter, Yue Wu, Ariel Rokem, and Aaron Y. Lee. "Deep-learning based, automated segmentation of macular edema in optical coherence tomography." Biomedical optics express 8, no. 7 (2017): 3440-3448.

[121] Yu, Kai, Fei Shi, Enting Gao, Weifang Zhu, Haoyu Chen, and Xinjian Chen. "Sharedhole graph search with adaptive constraints for 3D optic nerve head optical coherence tomography image segmentation." Biomedical optics express 9, no. 3 (2018): 962-983.

[122] L. Breiman, "Random forests," Mach. Learn. 45(1), 5-32 (2001).

[123] Varga, László, Attila Kovács, Tamás Grósz, Géza Thury, Flora Hadarits, Rózse Dégi, and József Dombi. "Automatic Segmentation of Hyperreflective Foci in OCT Images." Computer Methods and Programs in Biomedicine (2019). 
[124] Faust, Oliver, Yuki Hagiwara, Tan Jen Hong, Oh Shu Lih, and U. Rajendra Acharya. "Deep learning for healthcare applications based on physiological signals: A review." Computer methods and programs in biomedicine 161 (2018): 1-13.

[125] Ronneberger, Olaf, Philipp Fischer, and Thomas Brox. "U-net: Convolutional networks for biomedical image segmentation." In International Conference on Medical image computing and computer-assisted intervention, pp. 234-241. Springer, Cham, 2015.

[126] Sun, Yankui, Shan Li, and Zhongyang Sun. "Fully automated macular pathology detection in retina optical coherence tomography images using sparse coding and dictionary learning." Journal of biomedical optics 22, no. 1 (2017): 016012.

[127] Pilch, Matthäus, Knut Stieger, Yaroslava Wenner, Markus N. Preising, Christoph Friedburg, Erdmuthe Meyer zu Bexten, and Birgit Lorenz. "Automated segmentation of pathological cavities in optical coherence tomography scans." Investigative ophthalmology \& visual science 54, no. 6 (2013): 4385-4393.

[128] Fang, L., Jin, Y., Huang, L., Guo, S., Zhao, G. and Chen, X., 2019. Iterative fusion convolutional neural networks for classification of optical coherence tomography images. Journal of Visual Communication and Image Representation, 59, pp.327-333. 